\title{
Educación y políticas de la memoria sobre la historia reciente de América Latina*
}

Education and Memory Policies About Recent

History of Latin America

Educação e políticas da memória sobre a história recente da América Latina

\author{
Martha Cecilia Herrera* (iD orcid.org/0000-0003-4170-7822 \\ Carol Pertuz Bedoya** (iD orcid.org/0000-0003-2184-0933
}

\author{
Artículo de investigación \\ Revista Colombiana de Educación, N.ํㄱ. Segundo semestre de 2016, Bogotá, Colombia. \\ Para citar: Herrera, M. C., y Pertuz, C. (2016). Educación y políticas de la memoria sobre la \\ historia reciente de América Latina. Revista Colombiana de Educación, (71), 79-108.
}

Recibido: 12/01/16

Evaluado: $9 / 02 / 16$

$31 / 01 / 16$

* El artículo condensa uno de los capítulos de los resultados de la investigación Educación y políticas de la memoria en América Latina. Financiada por la Universidad Pedagógica Nacional para el período 2015-2016. Código DPG-418-15.

** Docente investigadora de la Universidad Pedagógica Nacional (Colombia). Doctora en Filosofía e Historia de la Educación de la Universidad Estadual de Campinas (Brasil). Fundadora del grupo de investigación Educación y Cultura Política. Coordinadora del proyecto "Narrativa testimonial, políticas de la memoria y subjetividad en América Latina" del Centro de Investigaciones de la Universidad Pedagógica (cIUP). Correo electrónico: malaquitalO@gmail.com

*** Licenciada en Psicología y Pedagogía de la Universidad Pedagógica Nacional (Colombia). Estudiante de la Maestría en Estudios en Infancias de la Universidad Pedagógica Nacional y la Universidad de Antioquia. Miembro del grupo de investigación Educación y Cultura Política. Correo electrónico: carolpertuz@gmail.com 


\section{Resumen}

El artículo presenta resultados investigativos en torno a las políticas de memoria sobre la violencia política acontecida en la historia reciente de América Latina y sus articulaciones con el campo de la educación. Se auscultan algunas de sus particularidades en Chile, Argentina y Colombia, distanciándose del paradigma del sujeto-víctima que ha pautado buena parte de las políticas de memoria oficiales.

\section{Palabras clave}

políticas de la memoria; políticas educativas; cultura política; América Latina

\section{Keywords}

memory policies; educational policies; political culture;

Latin America

\section{Abstract}

This article presents research results about memory policies concerning political violence in the recent history of Latin America, and their articulations with education. It explores some of their particularities in Chile, Argentina and Colombia, taking distance from the paradigm of victim-subject which has largely prevailed in official memory policies.

\section{Resumo}

O artigo apresenta os resultados da investigação acerca das políticas da memória sobre a violéncia política que ocorreu na história recente de América Latina e suas articulações com os domínios da educação. Auscultam-se algumas de suas particularidades no Chile, na Argentina e na Colômbia, afastando-se do paradigma do sujeito-vítima que pautou uma parte considerável das políticas de memória oficiais.

\section{Palavras chave}

políticas da memória; políticas educacionais; cultura política; América Latina 


\title{
Las sombras del pasado/presente: violencia política y pedagogías del miedo
}

\begin{abstract}
A abordar el análisis de la historia reciente de América Latina y los fenómenos de violencia política a ella asociados desde la década de los 70, una de las problemáticas que emerge, para el caso de Chile y Argentina bajo el peso de las dictaduras, y para el caso de Colombia bajo medidas de excepcionalidad en el marco de una democracia restringida, tiene que ver con las secuelas de la represión desplegada por el terrorismo de Estado en las subjetividades, expresada, entre otras, a través de la instauración de lo que muchos han nombrado como una "pedagogía del miedo" (Clara, 2008), una "cultura del miedo" (Acosta, 2001; Lechner, 1990; Rubio 2013), una "pedagogía de la sospecha" (Mariño, 2006), o el "miedo a la democracia" (Grupo de Memoria Histórica, 2013a) que penetró de diversas maneras en los intersticios del tejido social y afectó la esfera política y cultural, así como la cotidianidad y las formas como los sujetos se pensaban y se relacionaban, recomponiendo en varios sentidos las culturas políticas y afianzando algunos de sus componentes autoritarios, obturando, de paso, las posibilidades de participación política y el desarrollo del pensamiento crítico y de proyectos emancipatorios, pero sin impedir por ello la emergencia de intersticios que posibilitaron actos de oposición y resistencia.
\end{abstract}

En los debates adelantados en el parlamento chileno sobre la ley de reparación de víctimas, a un año de la entrega del Informe Rettig, el representante Roberto Muñoz Barra mencionaba el comienzo de un presente marcado por la instauración de los derechos humanos el cual impelía a someter a crítica el legado de un pasado modulado por la conculcación de estos derechos y por el despliegue de dispositivos pedagógicos fundados en el miedo. En sus palabras:

Se abre una nueva etapa en materia de derechos humanos para Chile; comienza el tiempo de la reflexión -que tanta falta nos hace-, del diálogo y de la construcción social de la verdad. De ello depende que cada uno de los chilenos nos libremos de los hábitos y conductas aprendidas en la pedagogía del miedo que impuso el gobierno autoritario, miedo que nunca deberá repetirse (Muñoz, citado por Lira y Loveman, 2005, p. 137).

Frente a este legado era necesario construir, en materia de política pública, una memoria para educar

a las futuras generaciones en valores tales que impidan que la fuerza triunfe sobre la razón, que el crimen sobre la vida, que la mentira sobre la verdad, que la impunidad sobre la justicia, la verdad debe constituirse en una de las fuerzas que guíe la conducta de la sociedad (Lira y Loveman, 2002, p. 158) 
Pilar Calveiro (1998) aludía, por su parte, a los efectos causados por la acción del terrorismo de Estado en Argentina al marcar la memoria colectiva con sentimientos asociados a la idea de "lo difícil que es sobrevivir a los embates de un poder autoritario y desaparecedor". Según ella: "La acción del terror no acabó el día que cayó el gobierno militar. Hay un efecto a futuro, un efecto que perdura en la memoria de la sociedad" (p. 98).

En el caso de Colombia, la persecución a la prensa independiente, el asesinato de líderes sindicales y otros luchadores sociales, así como la zozobra causada en poblaciones rurales y barrios urbanos periféricos, en donde incluso las escuelas se han convertido en fortín de los actores armados, guerrilleros, ejército, paramilitares, han quebrado los lazos de solidaridad y diseminado la falta de confianza entre sus moradores al imponer la ley del silencio entre los mismos, así como una cotidianidad marcada por el terror, lo cual alimenta las memorias individuales y colectivas en diversos territorios del país (Pecaut, 2001, p. 249).

Sobre la base de este tipo de panorama, las políticas públicas en los tres países han debido plantearse no solo cómo tramitar el legado del pasado, haciendo justicia a la memoria de las víctimas, de los ausentes y de los sobrevivientes (en los planos jurídico, económico y social), sino también cómo recomponer el tejido social y con ello las subjetividades arrasadas por los acontecimientos de violencia política, reto que es mayor en el caso de Colombia por encontrarse todavía en un contexto de conflicto armado. Tarea intergeneracional, ¿o transgeneracional?, que debe prestar atención a quienes vivieron los acontecimientos del pasado pero también a sus descendientes, quienes en medio de regímenes democráticos (los cuales cuentan con bastantes limitaciones) han heredado de sus ancestros un pasado turbulento cuyas marcas son el piso sobre el cual los nuevos regímenes se asientan, a quienes crecen (o crecerán) en medio de horizontes no del todo esclarecidos respecto a las vicisitudes en la configuración de órdenes sociales democráticos y sus posibilidades futuras de continuidad.

Resulta obvio que esta problemática no se reduce solo a los alcances que el miedo ha dejado en el tejido social, pero constituye, sin duda, una puerta de entrada, puesto que este tipo de tecnologías condensa aspectos a considerar cuando se piensa en las incidencias de la represión en las culturas políticas y en las formas de configuración de las ciudadanías en las sociedades contemporáneas. Si bien los regímenes represivos hicieron uso del miedo y su despliegue dejó numerosas secuelas en los sujetos, al mismo tiempo podemos preguntarnos cómo lo han canalizado los nuevos poderes estatuidos y/o han hecho uso de su evocación en el plano de la memoria colectiva para impulsar políticas, en las que, según lo postula Colin Robin (citado por Pincheira, 2010, p. 579) "el miedo pasa a constituirse como instrumento de un nuevo consenso moral y político"; y, del 
mismo modo, pasar a cuestionarnos por las líneas de fuga y de resistencia que emergieron en estos contextos con el propósito de modificar el orden social estatuido.

Desde este ángulo cabe, entonces, interrogarnos respecto a cómo se han venido dando las relaciones entre las políticas públicas de memoria y las políticas educativas en estos tres países para reflexionar sobre la problemática expuesta. En esta medida, en el presente artículo abordamos, en primer lugar, algunas consideraciones en torno a la cultura política y las políticas de memoria, para pasar después a señalar las características de tales políticas en Chile, Argentina y Colombia, así como sus intersecciones con el campo de la educación. Por último, a manera de conclusiones, planteamos algunas reflexiones en relación con la problemática expuesta.

\section{Culturas políticas y políticas públicas de memoria}

Como parte de los procesos de transición democrática o de procesos de legitimación institucional dados en varios países de América Latina, en el marco de las deudas en materia de gobernabilidad y de derechos ciudadanos heredadas por los regímenes empeñados en sofocar los movimientos de disidencia y desarticular los imaginarios políticos y sociales que aparecían como obstáculos a la consolidación de los modelos de desarrollo capitalista, en el marco de la Guerra Fría y de los contextos particulares de cada país, se emprendieron elaboraciones en torno a la formación política y a la construcción de culturas políticas de carácter democrático que pautaron los derroteros de las políticas públicas.

Desde este horizonte, se considera pertinente abordar el estudio de las prácticas relacionadas con la memoria de los hechos referidos a la violencia política en Chile, Argentina y Colombia, a los grupos y las fuerzas que las vehiculizan y las formas como se han posicionado en la arena pública, como parte de los repertorios que ayudan a configurar las culturas políticas, contribuyendo al análisis sobre los elementos de carácter simbólico que han incidido en ellas, así como al papel que ha tenido la memoria en cuanto matriz de significación de lo político y en la configuración de las subjetividades.

Las políticas en torno a la memoria de la violencia política en estos países visibilizaron diversos actores en el escenario público y, en el caso de las víctimas y las organizaciones que emprendieron la denuncia de los atropellos vividos, cuestionaron el orden institucional y las versiones oficiales acerca de lo acontecido. Estos actores incorporaron, a través de sus luchas, nuevas categorías interpretativas sobre la cultura política y ampliaron, en este sentido, las acepciones en torno a la democracia y a sus horizontes normativos. Según Pérotin Dumon: 
Los imperativos de verdad y memoria forman parte de la cultura política contemporánea de América Latina, en particular donde hubo un notable movimiento por defender los derechos humanos. Y en ese contexto, "verdad" y "memoria" forman en lo sucesivo un par y se asimilan una a otra (2005).

De este modo, las políticas de la memoria que alimentaron los reservorios de la cultura política a lo largo de estas décadas no solo se refieren a las políticas tramitadas por el Estado y, si bien, en ellas se expresan varias de las tensiones existentes sobre las formas de concebir el pasado y el orden social, lo cierto es que los usos políticos de la memoria recorren canales diversos y contribuyen a configurar variados relatos según las apropiaciones que hacen los sujetos y los colectivos en los cuales se ponen en juego distintos repertorios sociales, políticos y culturales. Así, esta serie de relatos y sus diversas significaciones sobre el pasado, con las consecuentes repercusiones sobre el presente y los horizontes de futuro, dan pie a diversas luchas por la memoria, dentro de las que se encuentran las pugnas por su representación en el campo de las políticas oficiales. Para Lechner y Güell (2006):

La lucha de las diferentes identidades colectivas por rememorar sus respectivas historias remite a un ámbito de representación donde reconocerse y ser reconocida. A su vez, las posibilidades y alcances de esa lucha están marcados por la forma y dinámicas de ese ámbito. La disputa de las memorias remite pues a la política en tanto "puesta en escena" de las memorias posibles. Toda sociedad posee una política de la memoria más o menos explicita, esto es el marco de poder dentro del cual (o contra el cual) la sociedad elabora sus memorias y olvidos (p. 22).

En este sentido los usos políticos de la memoria no pueden entenderse solo en términos de su expresión en las políticas oficiales sino como un proceso de configuración dado en diversos escenarios sociales y tramitado por distintas fuerzas que tensionan las expresiones de cultura política y disputan al Estado su voluntad de hegemonizar en torno a ellos. Desde este ángulo, Ricard Vinyes (2009) diferencia las categorías de memoria pública, política pública de memoria y memoria oficial. Para el autor la memoria pública se refiere a:

La imagen del pasado públicamente discutida, por lo que se construye en el debate político, social y cultural que produce la sociedad según cada coyuntura con la intervención de todos los agentes; y una de las funciones de la política pública es, precisamente, garantizar la participación de los diferentes actores en la confección de la memoria pública (p. 58).

De este modo, el ejercicio de la memoria pública deberá ser protegido por el Estado como un derecho, a través de una política pública 
de memoria que garantice su existencia y la expresión en ella de la pluralidad de memorias presentes en la sociedad, correspondiendo a los diversos actores pugnar por condiciones para el reconocimiento de sus puntos de vista en la esfera pública. Por su parte, la memoria oficial alude, de manera más restricta, al Estado y a sus intereses por hegemonizar y monopolizar la memoria pública. Es en el entrecruzamiento de estas tres aristas (memoria pública, política pública y memoria oficial) y la serie de dinámicas que se generan entre ellas que habría que entender las políticas de la memoria referidas a la violencia política que ha sacudido a América Latina desde las décadas de los 60 y 70, así como los aspectos asociados a su transmisión, apropiación y recreación por parte de los distintos sujetos y actores sociales en el marco de las sociedades contemporáneas, aspectos que, a su vez, nos permiten entender algunas de las dinámicas de construcción de la cultura política. Veamos cómo se han dado estas dinámicas en los tres países foco de nuestra investigación.

\section{Las políticas públicas de memoria sobre el pasado reciente y sus nexos con lo educativo}

Las políticas públicas implementadas por los gobiernos transicionales, en Chile y Argentina, así como en Colombia -en el marco de otro contexto político-, han acudido a variadas iniciativas en las cuales se ha dado importancia a diversos espacios de formación. En este sentido, tuvieron un despliegue importante a partir de 1990 en los dos primeros países y en la década del 2000 en Colombia. Estas políticas han estado caracterizadas por las diversas coyunturas de orden político y las formas como los gobiernos tramitaron el pasado; al mismo tiempo, estuvieron sujetas a los diferentes pulsos dados en el campo de la memoria pública entre los movimientos en defensa de los derechos humanos, las víctimas, así como los sectores que han hegemonizado en la política de memoria postulada desde el Estado.

En los lineamientos de las políticas han tenido gran repercusión los consensos logrados a partir de los informes de las comisiones de la verdad (Rettig y Valech en Chile y Conadep en Argentina), así hayan sido cuestionados algunos de sus hallazgos e interpretaciones al ser leídos a la luz de diversos intereses, de nuevos contextos históricos o del conocimiento de hechos que han obligado a su relectura. Por su parte, en el caso de Colombia, la Ley de Justicia y Paz de 2005 y la Ley de Víctimas de 2011 constituyen el referente de las políticas de memoria recientes, y el Informe ¡Basta Ya! Colombia: memorias de guerra y dignidad (2013), un logro importante en la búsqueda de comprensión sobre el conflicto armado.

En los tres países el referente de las políticas se basa en la idea de un orden social apoyado en un sistema político sustentado en la democracia 
representativa, en donde el sujeto ciudadano ( $y$, dentro de él, el sujeto-víctima) se entiende en clave de derechos, horizonte desde el cual se apunta a la consolidación de culturas políticas de carácter democrático. No obstante, en el complejo entramado que implica el establecer acuerdos entre las distintas fuerzas, actores y sujetos sociales, se encuentran en juego las acepciones en torno a la construcción de un orden democrático en el que los repertorios sociales, culturales y políticos, que estas fuerzas vehiculizan, arrojan diversas memorias de las que también se vale el orden dominante para interpelar y, en más de una ocasión, subyugar a sus gobernados. En este sentido, estas memorias deberán ser esclarecidas por el pensamiento crítico para posibilitar puntos de fuga y construcción de proyectos emancipatorios, pues como menciona Moulian:

Las razones de Estado juegan con la inocencia de los hombres comunes. Manipulan los espantapájaros del miedo para que la memoria triture los recuerdos. Para que los hombres comunes sientan hastío ante el recuerdo, que amenaza romper la paz cotidiana. Pero esos recuerdos bloqueados seguirán bajo la superficie realizando su daño sordo (1997, p. 42).

Veamos a continuación algunos de los rasgos que caracterizaron las políticas públicas en estos tres países.

\section{Políticas públicas de memoria en Chile y Argentina}

\section{Chile}

En Chile el proceso de transición hacia un régimen democrático comenzó desde la década de los 80, a través de una serie de acuerdos entre el gobierno militar y las fuerzas que retomarían la institucionalización de un nuevo orden social. Con este escenario restricto, la Concertación de Partidos por la Democracia (una coalición de partidos de izquierda, centroizquierda y centro) se comprometió a respetar la Constitución instaurada en 1980 por el gobierno militar y a garantizar la continuidad de Pinochet como jefe de las Fuerzas Armadas hasta 1988, año en el que este se hizo nombrar como senador vitalicio, con lo cual "trocaba su base militar de poder por la entrada oficial a la clase política del país" (Wilde, 2007, p. 4). En marzo de 1990 Patricio Aylwin, socialdemócrata, asumió la presidencia y se propuso esclarecer las violaciones a los derechos humanos bajo la dictadura, para lo cual se conformó la Comisión Nacional de Verdad y Reconciliación, que funcionó bajo condiciones limitadas. En palabras de Carretero y Borrelli: 
años 70. El miedo a revivir los conflictos llevó a encararlos a partir del lema de la reconciliación nacional. Aunque paulatinamente se aceptó públicamente la existencia de violaciones a los derechos humanos durante el régimen pinochetista, no se indagó en las responsabilidades. En ese sentido, más que una memoria del olvido se trató de erigir una memoria del silencio (2008, p. 211).

No obstante, una serie de acontecimientos dados en la década del 90 conmocionaron la esfera nacional y dieron pie a las "irrupciones de la memoria" (Wilde, 2007) que Ilevaron a abrir la caja de los recuerdos (Stern, 2000) y a que los movimientos sociales en contra del régimen pinochetista se reactivaran. Dentro de estos acontecimientos el más importante, sin duda, fue el arresto de Pinochet en Londres en el año de 1998, el cual dejó al descubierto la fuerza de las memorias en pugna y las dificultades por establecer consensos sobre el pasado reciente.

Al concentrarse en las víctimas desaparecidas, el Informe de la Comisión Nacional de Verdad y Reconciliación sobre la violación a los derechos humanos en Chile, 19731990 (1991) -conocido como el Informe Rettig)no abordó el tema de las víctimas sobrevivientes y los vejámenes a los cuales habían sido sometidas, lo cual condujo a presiones por parte de organismos de defensa de los derechos de las mismas con el fin de evitar el olvido y la impunidad pugnando, en este sentido, por la ampliación de la política de memoria oficial. En este entorno, el presidente Ricardo Lagos, socialista, dispuso en 2004 la conformación de la Comisión Nacional sobre Prisión Política y Tortura (conocido como el Informe Valech).

Respecto a los resultados de estas dos comisiones, Pérotin Dumon (2005) señala, en primer lugar, el carácter legitimador que tuvo ante la opinión pública el Informe Rettig, aunque este no contó con una difusión masiva, debido a las tensiones que se respiraban en el escenario político por presiones de los militares y sectores de ultraderecha. En segundo lugar, en contraste con el Rettig, el Informe Valech tuvo mayor resonancia, tanto por los alcances de la investigación Ilevada a cabo -al incluir en calidad de víctimas no solo a los desaparecidos sino también a los sobrevivientes, lo cual amplió su alcance en el plano sociológico-, como por el tiraje de la edición, que alcanzó los 33000 ejemplares. De este modo, los dos informes y más notoriamente el segundo, consiguieron obtener un consenso social propiciado desde el Estado, en torno a los acontecimientos relacionados con el periodo 1973-1990. En esta dirección, la presentación que en 2004 hacía el presidente Lagos sobre su política, bajo el título No hay mañana sin ayer, resaltaba la defensa de los derechos humanos como signo de identidad fundacional de la coalición de gobierno y como basamento para el fortalecimiento de una cultura política democrática.

Durante el primer mandato de Michelle Bachelet (2006-2010) se dio continuidad a esta política y se inauguró el Museo de la Memoria y los 
Derechos Humanos en Santiago (2010). En 2011, bajo la presidencia de Sebastián Piñera (primer presidente de tendencia derechista), se dio a conocer el informe de la Comisión Presidencial Asesora para la Calificación de Detenidos, Desaparecidos, Ejecutados Políticos y Víctimas de Prisión Política y Tortura, creada en 2009, conocida como Comisión Valech II. Esta comisión pretendía, en cierta medida, concluir el ciclo iniciado con la Transición en la búsqueda de verdad, justicia y reparación, como lo mencionó Piñera al afirmar la voluntad de que "el cierre de este proceso y el reconocimiento que realiza, contribuya a la consolidación de una sociedad chilena capaz de construir una convivencia democrática basada en la tolerancia y el respeto por los derechos humanos de cada persona" ( $\mathrm{La}$ Nación, 26 de agosto de 2011).

Para Jaume Peris la fórmula de reconciliación y consenso que prevalece en la política de memoria oficial Ilevó, entre otras, a que el informe valech se refiriera a los acontecimientos ocurridos bajo la dictadura en una clave retórica que los deshistorizó, al inscribirlos en una suerte de disfunción psicológica colectiva que eludía "la mirada de la productividad social de la violencia y de su rol en la constitución de la sociedad chilena actual" (2009, p. 72). Así, pese al énfasis del informe en las víctimas y su apoyo en los testimonios de los sobrevivientes, estas no contaron con un lugar de decisión ni de visibilidad en las políticas públicas, pues su presencia se tornaba incómoda frente a una actitud que prefería el olvido del pasado en lugar de una mediación histórica que lo tramitase. De este modo, al inscribir la violencia de Estado en el paradigma de los derechos humanos, bajo una idea omnicomprensiva de la violencia, se le despojó de su centralidad, siendo esta la misión que la comisión tenía asignada bajo su mandato (Peris, 2008, p. 279).

Por su parte, Nelly Richard (2010) interpela en sus escritos las políticas de memoria desplegadas a lo largo de estas décadas y señala como ellas encauzaron los usos del recuerdo a favor de una política de reconciliación nacional que invisibilizó las expresiones que "hacían ruido" a la idea del consenso puesto en marcha por los gobiernos de la Concertación. Para la autora, los principales hitos que delinearon la memoria pública e institucional en Chile pueden ser nombrados de la manera siguiente:

La secuencia de la memoria pública e institucional de la transición combinó fechas y conmemoraciones (el 11 de septiembre de 1973, los 30 años del golpe militar), Informes, Comisiones y Tribunales (el Informe Rettig, el Informe Valech, la Mesa de Diálogo); recordatorios y monumentos (el Parque por la Paz de Villa Grimaldi, el Memorial del Cementerio General, el Museo de la Memoria de los Derechos Humanos) para fijar así los usos del recuerdo en función del ideal reconciliatorio del consenso como modo de integración forzada de lo 
políticamente escindido, de lo socialmente desintegrado, a la plenitud de una comunidad dañada y luego curada en sus heridas por la moral del perdón (p. 17).

\section{Argentina}

A diferencia de Chile, donde se llevó a cabo un proceso de transición bajo un modelo que implicó pactos y compromisos con los militares, en Argentina el colapso del régimen marcado por fracasos en el modelo político-económico y por la derrota en la guerra con Inglaterra en torno a las Malvinas (1982) llevó a una transición no pactada. Así, desde 1981 se inició la consolidación de la Multipartidaria Nacional, una coalición de organizaciones que incluyó a la Unión Cívica Radical, el Partido Justicialista (peronista), el Intransigente, el Demócrata Cristiano, y el Movimiento Integración y Desarrollo, con el objeto de presionar a la Junta Militar a abandonar el poder y proceder a instaurar un régimen democrático. La Multipartidaria fue disuelta una vez logró su objetivo en 1983 cuando Raúl Alfonsín, de la Unión Cívica Radical, asumió como presidente electo.

Una vez instaurado el régimen democrático, Alfonsín procedió a crear la Comisión Nacional sobre la Desaparición de Personas (Conadep) para investigar los crímenes de la dictadura militar entre 1976 y 1983. El informe de la Conadep sirvió de fundamento para la elaboración del Nunca más -también conocido como Informe Sábato-. En este informe se describió la suerte de unos 9000 casos de desapariciones (si bien en el imaginario social se afianzó la idea de 30000), se reconstruyeron los métodos de la maquinaria represiva y se incluyó una selección de testimonios sobre la dictadura (Crenzel, 2008, 2010; Vezzetti, 2007). Este informe fue uno de los insumos en los que se apoyó el Juicio a las Juntas que tuvo lugar en 1985, y se consolidó como relato oficial que permitió esclarecer el terrorismo de Estado como política sistemática, contexto en el cual se juzgó y condenó tanto a los militares integrantes de las Juntas como a los cabecillas de los grupos guerrilleros (Montoneros y ERP, la mayoría de ellos en calidad de reos ausentes). No obstante, el juicio a los militares presentó mayores repercusiones sobre la opinión pública.

El informe tuvo acogida inmediata debido "a la credibilidad de la voz de la conducción del Estado en la democracia recién recuperada y al prestigio de los miembros de la Comisión que, como sus portavoces, legitimaron su contenido" (Crenzel, 2008, p. 131), lo cual no quiere decir que no haya dado pie a diversas interpretaciones, debates y apropiaciones por los diferentes sectores de la sociedad. Los datos que verifican su éxito editorial, como uno de los indicadores de su amplia acogida, son descritos por Crenzel de la manera siguiente: 
El Nunca Más rápidamente se proyectó en la escena pública y obtuvo eco en la sociedad al convertirse en un éxito editorial. Su primera edición, en noviembre de 1984, de 40.000 ejemplares, se vendió en dos días. Entre esa fecha y marzo de 1985, además de editarse en idioma Braille, se vendieron 190.000 ejemplares del informe, incluyendo la edición publicada por el Círculo de Lectores y la primera editada en el exterior conjuntamente por EUDEBA y la española Seix Barral. Esta cifra representa el 38\% de los 503.830 ejemplares del Nunca Más que, como se dijo, se publicaron hasta noviembre de 2007, incluidas sus traducciones (Crenzel, 2008, p. 131).

Las presiones ejercidas por los militares, así como sectores de la derecha que tenían algún tipo de compromiso con lo ocurrido entre 1976 y 1983, condujeron -transcurrida la década- a la emanación de las leyes Punto Final (Ley 23492 sancionada en 1986) y Obediencia Debida (Ley 23521 sancionada en 1987), que paralizaron los procesos iniciados con el Juicio a las Juntas, proceso que estuvo acompañado por indultos ocurridos entre 1989 y 1990 bajo la presidencia de Menem, disposiciones legislativas que Ilegaron a ser conocidas como las leyes de impunidad. De acuerdo con Rosemberg y Kovacic (2010) "Estos cierres institucionales se vieron fortalecidos por una 'política de reconciliación' que creyó posible consolidar la democracia a partir del olvido de los crímenes de lesa humanidad cometidos desde el propio Estado" (p. 15). No obstante, como hemos mencionado, las políticas de memoria obedecen a la correlación entre las distintas fuerzas implicadas en el propósito de poner en la agenda pública sus significaciones sobre el pasado y con ello elevarlas a disposiciones normativas. Así, si bien:

Hasta la derrota militar de la dictadura argentina en la guerra contra el Reino Unido por la posesión de las islas Malvinas, en junio de 1982, las denuncias de los crímenes fueron neutralizadas [,] [t]ras la guerra, los organismos de derechos humanos canalizaron el repudio al régimen y se erigieron en un actor difícil de soslayar en la esfera pública (Crenzel, 2010, p. 6).

La década de los 90 dio paso a nuevos trabajos que enriquecieron el campo de la memoria pública y visibilizaron la militancia de muchas de las víctimas, así como las búsquedas identitarias de sus hijos. La década del 2000 en el marco de los gobiernos de Néstor Kirchner y de Cristina Fernández de Kirchner situó en la esfera pública el debate en torno a los años 70, al tiempo que convirtió los derechos humanos en política de Estado. Durante este periodo se declararon nulas las leyes de Punto Final y Obediencia Debida y se reanudaron los Juicios a las Juntas. Así mismo, los movimientos en defensa de las víctimas encontraron acogida en estas políticas y dieron su respaldo al Estado, aspecto que, a su vez, va a estar marcado por numerosas tensiones. 
En lo referente a las memorias que han sido instituidas por las fuerzas armadas en torno a lo acontecido en el periodo de la última dictadura es de destacar la consigna "Memoria completa", que fue cobrando cuerpo desde los 90 y a través de la cual se ha pretendido situar en calidad de sujetos/víctimas a los militares que murieron como fruto de los enfrentamientos dados entre las fuerzas armadas y las organizaciones guerrilleras en el periodo. Según Valentina Savi (2016):

\section{La consigna "Memoria Completa" reforzaba la imagen de una institución victimizada y damnificada por la violencia del pasado y relativizaba y obliteraba lo actuado por los cuadros del ejército durante el terrorismo de Estado. De este modo, el ejército se presentaba como una víctima más de la violencia de los $70^{\prime}$ (24 de marzo).}

Respecto al posicionamiento de esta memoria, Paula González menciona que si bien "desde mediados de los años noventa se contraponen la narrativa de la 'reconciliación nacional' con la reivindicación-visualización de la militancia revolucionaria, desde 2003 se confronta la institucionalización de la memoria -anclada en la relectura del Nunca más- con la 'memoria completa'" (2014, p. 57).

Aunque la adopción como política estatal de buena parte de las reivindicaciones de los organismos de derechos humanos durante los gobiernos kirchneristas logró dar continuidad a los procesos sobre verdad, justicia y reparación, y significó avances importantes a lo largo de doce años de gobierno, esto ha traído a su vez conflictos entre los diversos actores que interactúan en el campo de las políticas de memoria, en especial en lo relativo a la autonomía de algunas expresiones y a las tentativas oficiales de canalizar las iniciativas a favor de sus propios intereses.

No obstante, este panorama es susceptible de ser modificado a futuro, pues en las elecciones de 2015 Mauricio Macri -un empresario y dirigente deportivo, perteneciente al Partido Propuesta Republicana (PRO) de tendencia liberal conservadora- obtuvo la presidencia a través de la coalición Cambiemos. Macri ha manifestado su distanciamiento de las políticas kirchneristas, situación que tiene en actitud de alerta a diversos sectores de la sociedad, entre ellos a los organismos de derechos humanos y de defensa de las víctimas del terrorismo de Estado, quienes pugnan por garantizar la continuidad de las medidas de verdad, memoria y justicia en marcha. El día de su posesión Macri criticó "Ios fanatismos que nos arrastraron a la violencia", aseguró que luchará "contra la corrupción" al tiempo que abogó por una "justicia independiente que se limpie de un proceso de politización". En sus palabras: "No puede haber jueces militantes" (10 de diciembre de 2015, p. 12). 


\section{Algunos aspectos comunes en las políticas de memoria en Chile y Argentina}

Buena parte de la memoria social y sus expresiones en la memoria pública sobre las dictaduras militares en Chile y Argentina, han corrido en paralelo con la defensa de los derechos humanos y la demanda de justicia con sus diversos clivajes en las diferentes coyunturas políticas, siendo apuntaladas en torno al ideario de una cultura política democrática cuyas concreciones han dependido del pulso entre las fuerzas interesadas en dichos procesos y los alcances y significaciones que le han dado a dichos mandatos.

En relación con Argentina, Vezzetti se refiere a la emergencia de una narrativa estructurada bajo la adhesión al nuevo orden institucional de carácter democrático que desplazó a las significaciones anteriores, así como a la presencia progresiva del paradigma de las víctimas como pieza clave en las representaciones sobre el pasado de la dictadura, así como del testimonio como uno de sus relatos fundantes (2008, pp. 4-13). Para Crenzel (2009) y Marchesi (2011) los informes de las comisiones de la verdad tanto en Chile como en Argentina están atravesados por una narrativa humanitaria apoyada en los derechos humanos, la cual se gestó con anterioridad en el propio seno de los organismos de derechos humanos para viabilizar las denuncias de los crímenes cometidos por los militares, narrativa en la cual el reconocimiento de las identidades políticas de las víctimas pasó a un segundo plano por consideraciones que, en primera instancia, obedecieron a posiciones tácticas para habilitar lugares que posibilitaran su enunciación. Este aspecto nos permite vincularlas al paradigma del sujeto-víctima que se impuso en el ámbito internacional y que enfatiza en el perdón y en la reconciliación.

Marchesi sostiene, además, que los informes en Chile y Argentina privilegiaron argumentaciones referentes a polarizaciones ideológicas para explicar las causas de los golpes militares, sin entrar a considerar aspectos atinentes a factores sociales y políticos, como sí lo hicieron los informes de Brasil y Uruguay. El rechazo que en ellos se dio tanto a la violencia política como a "todo tipo de polarización y antagonismo entre miembros de la comunidad nacional", pretendía "ganar adhesiones y legitimidad en aquellos sectores del centro y la derecha más ambivalentes en las políticas de derechos humanos". Para el autor:

Esa percepción del antagonismo como algo peligroso para la estabilidad democrática limitó la acción de los movimientos sociales que mantenían críticas más radicales hacia el carácter de la transición, inclusive el propio movimiento de derechos humanos. En gran medida, la historia contada por los "Nunca Más" se presentaba como una parábola que advertía acerca de los riesgos que el desarrollo de políticas radicales podía generar en democracia (p. 22). 
En la actualidad, al tiempo que las políticas de memoria de carácter oficial en Chile y Argentina promueven una mirada con énfasis en la reconciliación, mucho más marcada en el caso chileno, lo cierto es que las diversas versiones del pasado reciente disputan los sentidos sobre los fenómenos históricos y han encontrado canales de circulación más expeditos que los proporcionados en tiempos de dictadura, cuando estaban obturados prácticamente todos los canales de opinión en los ámbitos nacionales. De esta manera, alimentan significaciones diferentes o distantes de las oficiales y contribuyen a gestar memorias de carácter múltiple en las que están en juego apuestas y utopías en torno al orden social, no solo con relación al pasado, sino también al presente y, en esta medida, con sus posibilidades de proyección hacia el futuro. Veamos, a continuación, de qué manera se han expresado estas políticas de memoria en el marco de las políticas educativas en estos dos países.

\section{Políticas de la memoria y enseñanza de la historia reciente en Chile y Argentina}

En consecuencia con lo anteriormente planteado, tanto en Chile como en Argentina las políticas educativas apropiaron la idea de los derechos humanos como el basamento de la cultura política democrática que debía ser consolidada por los nuevos órdenes sociales. Así lo expresaba en Chile el documento No hay mañana sin ayer del presidente Lagos:

La formación de nuestros niños y jóvenes de educación básica y media en los contenidos fundamentales de los derechos humanos y de la convivencia democrática constituye un elemento esencial para fortalecer la cultura política, social y moral de toda sociedad (2003, p. 31).

De manera general, las políticas de memoria muestran dos tipos de tratamiento sobre el pasado reciente en el currículum nacional de historia, uno de ellos, anterior a 1997, en el que no hay referencias al periodo de la dictadura, y el otro a partir de la reforma educativa de 1997, en el que este se aborda con base en la narrativa de la memoria emblemática pautada por el Informe Retting y, en general, por el desiderátum del ¡Nunca Más!. Según Carretero y Borrelli:

Los programas de estudio elaborados por el Ministerio de Educación en 1999 abonaron la idea de la "inevitabilidad" del golpe de Estado, sin adjudicarle responsabilidad directa a las Fuerzas Armadas en el derrocamiento de Allende. Sus Contenidos Mínimos Obligatorios no indicaban que debía estudiarse el periodo dictatorial, sino comparar el modelo económico que estableció el régimen con el existente previamente a septiembre de 1973. Justamente, se enfatizaba en los "logros" de la economía neoliberal del gobierno de Pinochet poniendo poca 
atención sobre sus efectos sociales [...]. Por su parte, los libros de texto aprobados y distribuidos gratuitamente por el Ministerio de Educación no mencionaban las palabras "golpe" ni "dictadura". Y se orientaban a comprender el golpe de 1973 como un desenlace inevitable de un conflicto "irresoluble" entre dos bandos que antes que transigir estimaban necesaria la lucha armada (2008, pp. 211-212).

Los debates sobre las reformas curriculares emprendidas a partir de 2006 evidencian las tensiones existentes entre las diversas fuerzas sociales respecto a la significación de la Dictadura Militar y a la inclusión de este periodo en los planes de estudio, contexto en el que varios sectores cuestionaron la viabilidad de "estudiar la transición a la democracia por carecer de la distancia temporal suficiente para mirar con objetividad el pasado reciente" (Reyes, 2013, pp. 44-45). Según Rubio (2013) se puede hablar de tres momentos que muestran diferentes articulaciones entre las políticas educativas y el tratamiento dado al pasado reciente a lo largo de estas décadas:

El período 1990-1996, en que se realiza una modificación de programas de formación ciudadana y de enseñanza de la historia, hecho que también está afectado por los cambios en los sentidos de la reforma educativa. Se trata de un período en que la orientación de modernización se sustenta en un fundamento comunitario-social. El segundo, entre 1996-2006, en que la modernización gira hacia los principios de la calidad y la equidad y se ordena desde el tecnicismo y el rendimiento sin modificar el tratamiento del pasado reciente, diagnosticando problemas en la formación ciudadana (Comisión 2004), sin realizar una discusión pedagógica sobre el sentido de los procesos formativos; y el período 2006-2010, en el cual se desarrollan ajustes curriculares aun en marcha y el pasado reciente es valorado como espacio para fortalecer una formación ciudadana en crisis, en aras a consolidar la democracia construida desde los 90 (pp. 385-386).

Para Reyes, las reformas curriculares aunque mencionaban incluir el punto de vista de los jóvenes en los programas de estudio y propiciar espacios que habilitaran el diálogo entre maestros y estudiantes, "la búsqueda de claves interpretativas que concordaran con el carácter de la transición democrática pactada" Ilevó a que se dejara en segundo plano "el conocimiento de los procesos sociales que explican los conflictos del presente" (p. 84), lo cual es un inconveniente puesto que el tratamiento de tales conflictos, al tener una mayor consonancia con las necesidades y expectativas de las actuales generaciones, permitiría establecer el puente histórico y los vínculos generacionales requeridos para una mejor comprensión sobre el presente.

En relación con Argentina, los temas que entraron en la agenda de la política educativa en el primer periodo señalan también el interés por 
fortalecer las instituciones como garantía de consolidación del nuevo orden social bajo el derrotero de los derechos humanos. Esta agenda es estudiada por Carbone teniendo en cuenta el análisis de "las prácticas relevadas del terreno de las prescripciones curriculares, de los movimientos sociales que tuvieron presencia en la reformulación de lineamientos de la educación pública y del escenario escolar como territorio del currículum real" (2012, p. 18). Estudio con base en el cual la autora indica la presencia en la política educativa de "las tensiones entre las herencias autoritarias y las vocaciones democráticas que enfrentaban a diversos actores representativos de poderes de decisión" (p. 17).

El informe Nunca Más fue incorporado como texto de estudio en las instituciones educativas, inicialmente en la Ciudad de Buenos Aires, a lo cual se agregó el que la editorial Eudeba hubiese solicitado a Dussel, Finocchio y Gojman, la elaboración de un texto escolar que tramitase pedagógicamente un informe denso y bastante crudo en algunas de las descripciones sobre las modalidades del terrorismo de Estado. Fue así que el texto Haciendo memoria en el país de Nunca Más (1997), trabajó los asuntos de la dictadura desde cuatro ejes de análisis que abarcaron el papel del Estado, los jóvenes, la violencia y la tolerancia, la memoria y el olvido. En él se procuró dotar de significación histórica los acontecimientos a los que aludía el Nunca Más. La primera edición del libro alcanzó un tiraje de 3000 ejemplares y "pese al alcance local de la ordenanza, el Ministerio de Educación dispuso su distribución en todo el país" (Crenzel, 2008, p. 166).

Si bien, como lo mencionan Carnovale y Larramendy, en términos generales "las decisiones en torno a políticas educativas estuvieron orientadas a promover el estudio del pasado reciente, en una clara voluntad de transmisión de valores democráticos, y de respeto y promoción de los derechos humanos" (2010, p. 241), se han presentado problemas en su implementación debido, por un lado, a las distintas coyunturas políticas que dieron determinados énfasis a las políticas de la memoria; $y$, por otro lado, a que las disposiciones legislativas no estuvieron acompañadas de una adecuada formación de los docentes, aspecto que también es destacado por Gonzalo de Amézola (2011). Según María Paula González, los puntos álgidos de debate en torno a la actualización de los contenidos curriculares de la historia, en la perspectiva de tomar distancia de enfoques tradicionales, versaron sobre:

El pasado reciente versus el pasado lejano; las disciplinas o las áreas; la enseñanza de problemas o del continuum histórico; el saber y el saber hacer; el lugar que ocupan los hechos, los conceptos y los procedimientos interpretativos e investigativos; el espacio para lo local en la enseñanza de las ciencias sociales (2014, p. 44). 
Para Carretero y Borrelli, aunque en Argentina se ha complejizado en la enseñanza la comprensión del pasado reciente, persisten problemáticas relativas a la memoria oficial y los riesgos de que esta invisibilice la pluralidad de expresiones de la memoria social y de la memoria pública:

El enfoque actual sobre la dictadura militar tiende a rebatir la "teoría de los dos demonios" de los primeros años democráticos (que igualaba la responsabilidad de las organizaciones armadas con el Estado militar y eximía de las propias a la sociedad civil) y las explicaciones reponen la complejidad del contexto histórico-político de los años 70. Sin embargo, aparecen otros problemas como la configuración de una "memoria oficial" sobre cómo recordar la dictadura militar, más aún cuando desde el año 2003 existe una política oficial del Estado que ha instalado la necesidad del recuerdo (p. 213).

De manera general, en el campo educativo la implementación de contenidos curriculares así como del desarrollo de prácticas educativas y pedagógicas en torno al pasado reciente, ha encontrado diferentes dinámicas en las instituciones educativas tanto en Chile como en Argentina en las que han estado en juego las características de la cultura escolar y sus desarrollos específicos, en los cuales se despliegan diversas tensiones entre los contenidos historiográficos y sus formas de adecuación y apropiación en los contextos escolares, con las luchas por la memoria que son vehiculizadas por los distintos sujetos que interactúan en el espacio escolar, así como las prácticas concretas, específicas, que se llevan a cabo en los establecimientos educativos. En términos generales, como ya lo mencionamos al comienzo, en los dos países las narrativas que más se han afianzado en los contextos educativos se apoyan en los informes de las comisiones de verdad histórica, a través de los cuales se logró un consenso en torno al repudio moral de los hechos acaecidos durante la dictadura, aunque en la actualidad dichas narrativas entran en confrontación con otros relatos y comprensiones que se han venido visibilizando en cada país, a partir de las distintas coyunturas históricas y que dan pie a múltiples tensiones.

Algunas de estas tensiones se trenzan alrededor de los ejes Historia y Memoria y la legitimidad de los saberes que encarnan cada uno de ellos con relación a las formas de comprensión del pasado reciente, tensiones en torno a las cuales entran en fricción referentes proporcionados por la Historia como disciplina, así como otros saberes de las ciencias sociales, además de las experiencias vividas por los docentes sobre este pasado (constituido en el momento como memoria viva), y los diversos posicionamientos de carácter ético y político sobre el período dictatorial y sus incidencias sobre el presente y el futuro que son vehiculizados por esta serie de fuerzas intervinientes. 


\section{Las políticas públicas de memoria en Colombia}

En Colombia, a partir de la Constitución de 1991, fruto de un interesante proceso de concertación entre distintas fuerzas sociales y políticas, dentro de las que se encontraban representantes de grupos guerrilleros reinsertados a la vida civil -Movimiento 19 de abril (M-19), una fracción del Ejército Popular de Liberación, EPL, y la corriente de Renovación Socialista, una disidencia del Ejército de Liberación Nacional, ELN, se estableció el Estado nacional como multicultural y se trazó el propósito de consolidar una cultura política democrática basada en el paradigma de los derechos humanos. Empero, la Constitución también trazaba los derroteros que permitirían acentuar el modelo económico neoliberal, al tiempo que el país atravesaba verdaderas crisis humanitarias fruto de la confrontación armada entre el Estado y el narcotráfico, así como de la irrupción a mayor escala del paramilitarismo, el cual arrasó, en más de una ocasión en connivencia con el ejército, poblaciones enteras con el lema de una lucha antiinsurgente en contra de las guerrillas de las Fuerzas Armadas Revolucionarias de Colombia (FARC) y el ELN. En la actualidad se están dando negociaciones con las Farc desde 2012 y se habla del inicio de una fase pública de negociaciones con el ELN.

La persistencia del conflicto iniciado desde las décadas del 60 y el 70 del siglo xx con el ciclo de violencia política conocida en el país como insurgente o revolucionaria -para diferenciarlo del ciclo anterior caracterizado por la violencia bipartidista (1948-1963)-, ha incidido en una serie de problemas en torno a la configuración de memorias sobre la violencia reciente $y$, como resultado, ha dado pie a la fragmentación de relatos que no logran mediaciones en el escenario público ni se articulan con narrativas más integradoras (no necesariamente unificadas). Una muestra de la fragilidad de estas memorias y de los escenarios cambiantes de los que se debe dar cuenta se expresa en las dificultades para precisar la temporalidad del conflicto actual, las causas que lo explican, las responsabilidades de los diferentes actores implicados, así como el tipo de víctimas a las que este ha dado lugar, de lo cual se desprenden, a su vez, los obstáculos para llegar a acuerdos sobre su solución y la implementación de políticas públicas de memoria y el logro de consenso social en torno al mismo.

A partir de la década del 2000 el tema de las víctimas empieza a ganar espacio en la agenda del Estado, al tiempo que se multiplican las acciones en torno a su memoria en variados escenarios sociales, lo cual ocurre en el contexto de iniciativas estatales que han buscado conseguir acuerdos con los grupos armados al margen de la ley. En este ámbito, durante el gobierno de Álvaro Uribe se expidió la Ley de Justicia y Paz (Ley 975 de 2005), con la cual se buscó propiciar la desmovilización de los grupos paramilitares creando la Comisión Nacional de Reparación y 
Reconciliación (CNRR). Esta comisión creó el Grupo de Memoria Histórica $(\mathrm{GMH})$ con el fin de emprender las investigaciones pertinentes para la comprensión del conflicto armado y poder derivar con base en ellas los ejes de las políticas públicas en torno a esta problemática.

La Ley de Justicia y Paz dejó un gran vacío en materia de víctimas lo cual condujo a la elaboración de una nueva ley, la Ley de Víctimas y Restitución de Tierras (Ley 1448 del 10 de junio de 2011), en el marco de la presidencia de Juan Manuel Santos. La ley estableció nuevas reglas respecto a los procesos de justicia y reparación y colocó en primer lugar a las víctimas, en consonancia con la actual tendencia internacional y en contraste con la Ley de Justicia y Paz que terminó visibilizando los intereses y la memoria de los victimarios. Por su parte el Grupo de Memoria Histórica fue adscrito al Centro Nacional de Memoria Histórica creado con motivo de la Ley 1448, con base en cuyas investigaciones se publicaron más de veinte informes parciales, así como un informe final denominado ¡Basta Ya! Colombia: Memorias de guerra y dignidad (2013a).

El ¡Basta Ya! logró consolidar estadísticas sobre las diferentes afectaciones del conflicto. Contabilizó un total de 220000 muertes entre 1958 y 2013, 25000 desapariciones forzadas y 5,5 millones de desplazados. Aunque sus resultados, especialmente en cifras, obtuvieron difusión en los principales periódicos del país, el informe no ha contado con una distribución masiva, e incluso es difícil encontrar en la página del Centro de Memoria Histórica información sobre el número de ejemplares difundidos o sobre los canales de circulación tanto de su versión completa (2013a) como de un resumen que se hizo de él (2013b).

Los diálogos emprendidos por el gobierno de Santos con las FARC desde el 19 de noviembre de 2012 han alcanzado notables desarrollos y en el momento actual hay gran expectativa de que se logre firmar un acuerdo de paz definitivo con esta organización en el transcurso del corriente año (2016). Por su parte, el pasado 30 de marzo se anunció el inicio de la fase pública de negociaciones de paz con el ELN, aunque todavía se está a la espera de la fecha de instalación de la mesa.

En el contexto de las conversaciones con las FARC, entre agosto de 2014 y febrero de 2015 se conformó una comisión de expertos denominada Comisión Histórica sobre el Conflicto y sus Víctimas (CHCV), cuya composición fue acordada entre el Estado y las FARC; la difusión sobre los resultados de su trabajo, según algunas opiniones, se ha restringido a los círculos intelectuales. Al mismo tiempo, se espera que una vez se firme un acuerdo entre las partes pueda constituirse una Comisión de la Verdad en torno a la cual existen divergencias de opinión por parte de sectores de oposición que desconfían de la legitimidad de dicha Comisión para representar los intereses del conjunto de la sociedad. La CHCV 
tampoco pudo llegar a un acuerdo respecto a los orígenes del conflicto, su periodización, las características de sus actores, ni de sus posibles soluciones, lo cual no posibilitó el consenso sobre la redacción de un documento único y llevó a la entrega de catorce textos firmados de manera individual. A mediano plazo, entre algunos sectores del Estado y de la sociedad civil se espera que a partir de una Comisión de la Verdad se pueda llegar a un relato canónico en torno al conflicto armado reciente, pero para esto se requiere que haya un cese del conflicto armado.

En este escenario, las actuales políticas de memoria acentúan la idea de un futuro inmediato caracterizado por el posconflicto en el marco de una cultura política democrática cuyos retos no logran ser ponderados en sus múltiples dimensiones, dentro de las cuales se incluyen el renacer de los grupos paramilitares, la débil intervención estatal en los territorios que dejarían los grupos guerrilleros, la importancia de políticas públicas que institucionalicen su presencia, la puesta en marcha de planes de desarrollo y reconstrucción del tejido social y la creación de una conciencia social y ciudadana en torno a los retos de la paz. Este último aspecto alude también a la importancia del establecimiento de vasos comunicantes entre las políticas de memoria estatal y las políticas educativas en torno a ellas. Veamos cuales han sido estas relaciones en las últimas décadas.

\section{Políticas de la memoria y enseñanza de la historia reciente en Colombia}

El diagnóstico sobre la crisis en la que se sumió el país desde la década del 70 -debido a la profundización de la violencia y a la violación sistemática de los derechos humanos, visibilizada en 1980 por la denuncia de Amnistía Internacional sobre el Estatuto de Seguridad del presidente Turbay Ayala (1978-1982)-, trajo aparejado un dictamen sobre la crisis de valores en la sociedad como una de las causantes de la violencia. Algunas de estas representaciones se difundieron con base en el informe de la Comisión de Violencia de 1987, el cual se refirió a una cultura de la violencia que debía encontrar solución con estrategias que promoviesen actitudes democráticas. Este dictamen condujo a la formulación de "un proyecto de formación ético-político para el país que permitiera transformar las conductas violentas de los ciudadanos", en el cual la escuela se identificó como escenario privilegiado (Cortés, 2012, p. 54, 55), lo cual condujo a la implementación de diversas iniciativas sobre educación ciudadana.

En 1984 en los planes de estudio de educación básica se fusionaron las asignaturas de Historia y Geografía, las cuales, en conjunto con otros saberes, como la Educación Cívica y la Economía, entre otros, pasaron a integrar el área de ciencias sociales. Por su parte, la Ley 115 de 1994, en concordancia con los lineamientos de la Constitución de 1991, trazó 
como uno de sus principales propósitos la consolidación de una cultura política democrática en las instituciones escolares e introdujo las figuras de personeros escolares nombrados por los mismos estudiantes, así como manuales de convivencia construidos a la luz de los proyectos educativos institucionales (Herrera, Pinilla, Díaz e Infante, 2005). Dicha ley nombró esta área como Ciencias Sociales, Historia, Geografía, Constitución Política y Democracia. Por su parte, los Lineamientos curriculares de Ciencias Sociales (2002) y los Estándares básicos en Ciencias Sociales (2004) establecieron derroteros en los que en el primer caso se enfatizaba en la enseñanza a partir de problemas y ejes generadores, mientras que en el segundo se daba prelación a los contenidos específicos por ciclo académico, lo cual propició confusión y debate para su aplicación por parte de los docentes (Acevedo y Samacá, 2012; Arias, 2015).

De este modo, los contenidos relacionados con la historia reciente encontraron espacios reducidos ya que, en buena parte, la insistencia en las metodologías en detrimento de una discusión profunda en torno a la actualización de los contenidos continuó privilegiando unidades temáticas que recordaban la historia tradicional y su sentido patriotero. No obstante, se abrió espacio a temas relacionados con el período conocido como la Violencia, referido a la segunda mitad del siglo xx, al tiempo que en los textos escolares de la década de 2000 se tematizaron problemáticas sobre la última mitad del siglo xx como movimientos sociales, conflictos políticos y sociales, identidad y memoria, así como tópicos que aluden a la violencia actual, la guerrilla, el narcotráfico, el paramilitarismo y los diálogos de paz (Arias, 2015).

En general, los textos escolares de ciencias sociales de la década del 2000 abordaron el tema del conflicto armado de manera tangencial y sin estrategias que posibilitaran incidencias en el plano cognitivo, en el desarrollo de un pensamiento crítico sobre la violencia política y el conflicto armado, o en la formación de ciudadanos democráticos. En la mayoría de los textos el conflicto armado se aborda, según Sánchez y Rodríguez, "mediante la descripción del desplazamiento forzado, del acuerdo humanitario y de la relación de la guerra interna con los derechos humanos y el derecho internacional humanitario" (2008, p. 204), pero no alcanzan a propiciar un pensamiento crítico respecto a dicha problemática.

En la presentación del Informe ¡Basta Ya! (2013a) se afirma que este "es un memorial de agravios de centenares de miles de víctimas del conflicto armado interno, pero también aspira a ser un acta de compromiso con la transformación del futuro de Colombia". Desde este horizonte, el informe indica la importancia de llevar a cabo un arduo trabajo de pedagogía política para lograr "una reconversión social" de lo que califica como un "escenario de tolerancias y complicidades con la guerra". Dicho trabajo 
de "reconversión social" deberá centrarse en posibilitar que se valore el disenso, la controversia, la diferencia como esencia de la democracia, difundiéndose de manera explícita el mensaje "de que las diferencias y la libertad de opinión e ideología son vitales en la consolidación de toda democracia y que el Estado las protege" (2013b, p. 14). Finalmente, el informe recomienda la adopción de los trabajos del Centro Nacional de Memoria Histórica y de los distintos emprendedores de la memoria en los proyectos institucionales expresados en "los currículos, proyectos pedagógicos, manuales y libros de historia y ciencias sociales" (p. 91). El propio CNMH tramitó una cátedra sobre el ¡Basta Ya! en 2014, en el marco de la Cátedra del Pensamiento Colombiano que tiene lugar en la Universidad Nacional, sede Bogotá, con el propósito de que la experiencia fuese replicada en otras universidades del país.

En el contexto del Informe iBasta Ya! y de las negociaciones que se adelantan en La Habana con las FARC, el Congreso expidió la Ley 1732, en septiembre de 2014, por la cual se estableció la Cátedra de la Paz "con el fin de garantizar la creación y el fortalecimiento de una cultura de paz en Colombia"; se fijó un plazo de seis meses para su puesta en marcha en los distintos establecimientos de formación. La cátedra deberá estar articulada con áreas del conocimiento: ciencias sociales como historia, geografía, constitución política y democracia; ciencias naturales y educación ambiental; educación ética y valores humanos. A este respecto, con base en las experiencias chilena y argentina, pueden verse los múltiples retos que tienen las instituciones escolares en materia de capacitación de docentes, adecuación de los currículos, renovación de las prácticas escolares, entre otros muchos aspectos, para dar tratamiento a las problemáticas relacionadas con la historia reciente y la formación de ciudadanos y de sujetos políticos capaces de incorporar de manera crítica los aprendizajes que de alli se puedan derivar.

Sandra Rodríguez (2014), al trazar un panorama sobre la enseñanza y el aprendizaje de las ciencias sociales entre 1990 y 2011, menciona que el debate acerca de esta problemática está a la orden del día y, según ella, "debe ser liderado por los investigadores y especialistas en el campo de la didáctica", ya que:

No se puede esperar a que lo colonicen las posiciones patrióticas que buscan un regreso a la historia decimonónica, ni las posiciones provenientes exclusivamente de la historia, que le atribuyen a la educación formal [...] la amnesia política que vive el país, como si los últimos 30 años no hubieran estado marcados por políticas de olvido e impunidad en diversos ámbitos de la vida nacional, que han incidido para que la historia reciente no se convierta en un objeto de debate público (p.141). 


\section{Conclusiones}

Como hemos visto, las políticas de memoria han delineado algunos de los ejes desde los cuales se ha pretendido incidir sobre las culturas políticas y la formación ciudadana en Chile, Argentina y Colombia desde la década de los 90 del siglo pasado, con el propósito de tomar distancia de las tradiciones autoritarias y de la imposición de pedagogías del miedo en la procura de consolidar órdenes sociales democráticos incluyentes. En general, las políticas con sus distintos clivajes en cada país han privilegiado el consenso y la reconciliación, lo cual ha llevado a invisibilizar aspectos sobre el pasado reciente y las responsabilidades de la sociedad en los hechos de violencia, al tiempo que han eludido la reflexión en torno a la productividad social de la misma en la constitución de las sociedades actuales. En esta medida, las secuelas del miedo a la amenaza política que marcó el terrorismo de Estado y otros fenómenos de violencia política, también han sido funcionales a las políticas de memoria oficiales en sus propósitos de eludir los conflictos derivados de memorias en pugna y de los intereses que estas vehiculizan, pues se considera que tales conflictos pueden fracturar las tentativas de una política de reconciliación, lo cual lleva a que las memorias no resueltas permanezcan fuera del escenario público o sean transfiguradas por la política oficial.

Así, desde el paradigma del sujeto-víctima que ha prevalecido en la memoria oficial impuesta en el escenario internacional desde la década del 60 -que cuenta con un marcado énfasis en la reconciliación- y ha tenido diferentes expresiones en los tres países, los sujetos afectados por la violencia se representan como individuos cuyas subjetividades se acotan al plano del sufrimiento, al tiempo que se diluyen sus biografías, sus vínculos políticos y sociales (Vinyes, 2009). Lo anterior, debido al tipo de apropiaciones que estas políticas han hecho del ideario de los derechos humanos al privilegiar el individuo como basamento de la cultura política y dar énfasis a lo jurídico y a los derechos negativos de los individuos frente al Estado, lo cual Ileva a negligenciar los nexos intersubjetivos inherentes a las relaciones humanas y a la configuración del orden social.

Al situarnos en un lugar distante de este paradigma, emerge la idea de pensar en los sujetos afectados por la violencia no solo desde su condición de víctimas, sino como sujetos portadores de proyectos e idearios que los habilitan como sujetos políticos cuyas trayectorias biográficas demandan ser situadas en el marco de una historia común. Esta perspectiva ha sido reivindicada en muchos de los casos por colectivos en defensa de los derechos de las víctimas, aunque también hay que señalar que el paradigma del sujeto-víctima encontró elaboraciones en el marco de estas agrupaciones en los periodos más agudos de la violencia estatal. Esta entrada permitiría activar un pensamiento crítico, un pensamiento histórico, 
que posibilite herramientas respecto a los usos políticos que los gobiernos en Chile, Argentina y Colombia le han dado al pasado conflictivo con base en memorias que eluden las responsabilidades éticas y políticas que el Estado y la sociedad han tenido sobre este pasado, y obturan la revisión de sus legados en el escenario educativo y en otros espacios sociales y culturales.

Lo anterior, señala la importancia de propender por una transmisión y apropiación del pasado por parte de la sociedad que propicie comunidades de memoria que sitúen los efectos de la violencia política no solo sobre sujetos o grupos específico sino también como asuntos que afectan al todo social, entendiéndolos como una herencia común que requiere ampliar la comprensión del nosotros afectado por la violencia como un universo restricto hacia el nosotros ciudadano. He ahí los múltiples retos que deberá abocar una pedagogía de la memoria sobre nuestra historia reciente.

Así mismo, las preocupaciones actuales que derivan de los diálogos de paz que se adelantan en Colombia pueden encontrar en los casos chileno y argentino una fuente de experiencias importante en el campo educativo -y en el impacto que en este han tenido las políticas de memoria- a través de las cuales es posible dimensionar las complejidades que entraña la reconstrucción del tejido social y la reinvención de nuestros modos de convivencia y respuesta a las demandas por la construcción de un orden social más incluyente. Así mismo, es preciso gestar escenarios que permitan garantizar interlocución en torno a los múltiples interrogantes de tipo pedagógico y ético-político -y curricular, si se quiere- que si bien han sido objeto de reflexión en el país durante las últimas décadas, hoy empiezan a ganar mayor visibilidad en distintos escenarios de formación.

En ese sentido, se torna necesaria una mirada no solo en relación con las políticas de memoria vinculadas al campo educativo sino con las distintas reflexiones de orden pedagógico generadas en los tres países en torno al sentido mismo de los procesos formativos, las cuales se relacionan con la necesidad de introducir en los espacios educativos las temáticas asociadas a los acontecimientos traumáticos del pasado reciente, también de reconocer la memoria no solo como contenido sino también estimar las distintas configuraciones que esta asume y sus relaciones con la cultura, las diferentes instituciones que le dan forma $y$, no menos importante, los diálogos que entabla con las actuales generaciones (Dussel, 2008). Estas responsabilidades pedagógicas, éticas y políticas tienen que ver con entender el recuerdo como "un proceso denso, cargado de múltiples dimensiones, donde intervienen sujetos e instituciones que imprimen sus propias huellas, mandatos, deseos" (p. 4); asimismo, implica contemplar los efectos en el largo plazo de las decisiones relacionadas con el qué, el cómo, el porqué y el dónde enseñar en relación con el 
recuerdo, pues, no todas las formas de recordar nos hacen avanzar hacia la construcción de memoria ni todas las maneras de hacer memoria conducen a las sociedades hacia procesos de reconciliación.

En última instancia, nos interesa resaltar la riqueza que puede entrañar una mirada que nos permita superar una tendencia autorreferencial y nos posibilite, al mismo tiempo, observar las confluencias de historias compartidas en Latinoamérica. Comprendernos en nuestra singularidad como país, pero también situarnos en una perspectiva latinoamericana. 


\section{Referencias bibliográficas}

Acevedo, Á. y Samacá, G. (2012). La política educativa para la enseñanza de la historia de Colombia (1948-1990): de los planes de estudio por asignaturas a la integración de las ciencias sociales. Revista Colombiana de Educación, (62), 219-242.

Acosta, Y. (2001). La estructura cultural de la contrarrevolución burguesa y los límites de las transiciones democráticas en el Cono Sur de América Latina. Utopía y Praxis Latinoamericana, 6 (12), 9-31.

Amézola, G. (2011). Historia enseñada e historia investigada: relaciones peligrosas. El tratamiento escolar de la última dictadura militar y la necesidad de una actualización académica en la formación de profesores. PolHis, 8, 9-26.

Arias, D. (2015). La enseñanza de las ciencias sociales en Colombia: lugar de las disciplinas y disputa por la hegemonía de un saber. Revista de Estudios Sociales, 52, 134-146.

Calveiro, P. (1998). Poder y desaparición. Los campos de concentración en Argentina. Buenos Aires: Colihue.

Camacho, Fernando. (2008). Memorias enfrentadas. Las reacciones a los informes Nunca Más de Argentina y Chile. Persona y Sociedad, (27), 67-99.

Carbone, G. M. (2012). Manuales de educación primaria en la Argentina (1984-1993). De las políticas soñadas a los proyectos inconclusos. En C. Kaufmann (coord.). Textos escolares, dictaduras y después. Mirada desde Argentina, Alemania, Brasil, España e Italia (pp. 17-50). Buenos Aires: Prometeo.

Carnovale, V. y Larramendy, Alina. (2010). Enseñar la historia reciente en la escuela: problemas y aportes para su abordaje. En Isabelino Siede. (Coord.) Ciencias sociales en la escuela (pp. 239-267). Buenos Aires: Aique.

Carretero, M. y Borrelli, M. (2008). Memorias recientes y pasados en conflicto: ¿cómo enseñar historia reciente en la escuela? Cultura y Educación, 20 (2), 201-215. Recuperado de: http://www.ub.edu/histodidactica/images/documentos/pdf/carretero-borrelli.pdf.

Clara, M. (2008). El sistema represor en la búsqueda de la pedagogía del miedo. Objetivos y consecuencias psicológicas del terrorismo de Estado. [En línea]. Recuperado de: http://www.psicologos.org.ar/docs/ MIRTA\%20CLARA\%20\%202008\%20EI\%20\%20sistema\%20represor $\% 20$ en $\% 20 l a \% 20 b \% C 3 \% B A s q u e d a \% 20 d e \% 20 l a \% 20 p e d a g o g \%-$ C3\%ADa\%20del\%20miedo.doc.

Comisión Nacional sobre la Desaparición de Personas (CONADEP). (1984). Nunca más. Informe de la Comisión Nacional sobre la Desaparición de Personas. Buenos Aires: Eudeba. 
Comisión Nacional de Verdad y Reconciliación. (1991). Informe Comisión Nacional de Verdad y Reconciliación (Rettig). Santiago: Corporación Nacional de Reparación y Reconciliación.

Comisión Nacional sobre Prisión Política y Tortura. (2004). Informe Comisión Nacional sobre Prisión Política y Tortura (Valech). Santiago: Gobierno de Chile, Ministerio del Interior.

Comisión Presidencial Asesora para la Calificación de Detenidos Desaparecidos, Ejecutados Políticos y Víctimas de Prisión Política y Tortura. (2011). Informe Valech II. Recuperado de: http://www.indh.cl/ informacion-comision-valech.

Cortés, R. A. (2012). Prácticas de ciudadanización en la escuela contemporánea. Colombia, 1984-2004 (tesis doctoral). Universidad Distrital Francisco José de Caldas, Universidad Pedagógica Nacional y Universidad del Valle. Bogotá, Colombia.

Crenzel, E. (2008). La historia política del Nunca Más. La memoria de las desapariciones en la Argentina. Buenos Aires: Siglo xxı.

Dussel, I. (2007). A 30 años del golpe: Repensar las políticas de la transmisión en la escuela. En G. Ríos, (ed.). La cita secreta. Encuentros y desencuentros entre educación y memoria (pp. 157-178). Santa Fe: Amsafe.

Dussel, I.; Finocchio, S. y Gojman, S. (1997; 2006). Haciendo memoria en el país del Nunca Más. Buenos Aires: Eudeba.

Gobierno de Chile. (2003). Propuesta del presidente Ricardo Lagos en materia de derechos humanos. No hay mañana sin ayer. Santiago: Autor. Recuperado de: http://www.ddhh.gov.cl/filesapp/propuesta_ DDHH.pdf.

González, M. P. (2014). La historia reciente en la escuela. Saberes y prácticas docentes en torno a la última dictadura. Buenos Aires: Polvorines.

Grupo de Memoria Histórica. (2013a) ¡Basta Ya! Colombia: Memorias de guerra y dignidad. Bogotá: Imprenta Nacional.

Grupo de Memoria Histórica. (2013b) ¡Basta Ya! Colombia: Memorias de guerra y dignidad. Resumen. Bogotá: Imprenta Nacional.

Herrera, M. C.; Pinilla, A.; Díaz, C. e Infante, R. (2005). La construcción de cultura política en Colombia: proyectos hegemónicos y resistencias culturales. Bogotá: Universidad Pedagógica Nacional.

Kaufmann, C. (coord.). (2012). Textos escolares, dictaduras y después. Mirada desde Argentina, Alemania, Brasil, España e Italia. Buenos Aires: Prometeo.

La Nación. (2011, agosto 26). Comisión Valech: Publicadas las listas con los casi 10 mil nuevos casos de DDHH. Recuperado de: http:// www.lanacion.cl/comision-valech-publicadas-las-listas-con-los-casi-10-mil-nuevos-casos-de-ddhh/noticias/2011-08-26/140725.html 
Lechner, N. (1990). Los patios interiores de la democracia. Subjetividad y política. Santiago: Fondo de Cultura Económica.

Lechner, N. y Güell, P. (2006). Construcción social de las memorias en la transición chilena. En E. Jelin; S.G.Kaufman (comps.). Subjetividad y figuras de la memoria (pp. 17-46). Buenos Aires: Siglo xxı.

Lira, E.; Loveman, B. (2002). El espejismo de la reconciliación chilena. 1990-2002. Santiago: LOM.

Lira, E.; Loveman, B. (2005). Políticas de reparación. Chile 1990-2004. Santiago de Chile: LOM.

Marchesi, A. (2011). El pasado como parábola política. En Badaró y Forné (eds.). Memorias de la represión en Argentina y Uruguay: narrativas, actores e instituciones (pp. 9-26). Sweden: Institute of Latin American Studies, Stockholm University.

Mariño, M. (2006). Las aguas bajan turbias. Política y pedagogía en los trabajos de la memoria. En Pablo Pineau (et ál.). El principio del fin. Políticas y memorias de la educación en la última dictadura militar (1976-1983) (pp. 119-210). Buenos Aires: Colihue.

Moulian, T. (1997). Chile actual. Anatomía de un mito. Santiago de Chile: LOM.

Página12. (2015, diciembre 10). Tenemos una visión nueva de la política. [En línea]. Recuperado de: http://www.pagina12.com.ar/diario/ ultimas/20-287960-2015-12-10.html

Pecaut, D. (2001). Guerra contra la Sociedad. Bogotá: Planeta.

Peris Blanes, J. (2008). Historia del testimonio chileno. De las estrategias de denuncia a las políticas de memoria. Valencia: Anejos de Quaderns de Filologia.

Peris Blanes, J. (2009). Contradicciones del testimonio. Políticas de la memoria y retóricas de la violencia en el Chile postdictatorial. Pasajes, 28, 71-79.

PérotinDumon, A. (2005). El pasado vivo de Chile en el año del Informe sobre la Tortura. En Nuevo Mundo Mundos Nuevos. DOI: 10.4000/ nuevomundo.954.

Pincheira Torres, I. (2010). Colin Robin. El miedo. Historia de una idea política. Polis (Santiago), 9(25), 577-581.

Reyes, L. (2013). A cuarenta años del golpe de Estado. El debate curricular inacabado. Docencia, 50, 30-46.

Richard, N. (2010). Crítica de la memoria (1990-2010). Santiago de Chile: Ediciones Universidad Diego Portales.

Rodríguez, S. (2014). Enseñanza y aprendizaje de la historia en Colombia, 1990-2011. En S. Plá, J. Pagès. La investigación en enseñanza de la historia en América Latina (pp. 109-154). México: Bonilla Artigas-Universidad Pedagógica Nacional. 
Rosemberg, J.; Kovacic, V. (2010). Educación, memoria y derechos humanos: orientaciones pedagógicas y recomendaciones para su enseñanza. Buenos Aires: Ministerio de Educación de la Nación.

Rubio, Graciela. (2013). Memoria, ciudadanía y lo público en la elaboración del pasado reciente en la experiencia chilena. Memoria y Sociedad, 17 (35), 164-183.

Sánchez, O. M. y Rodríguez, S. P. (2008). Narrativa, memoria y enseñanza del conflicto armado colombiana: propuesta para superar las políticas de olvido e impunidad. En D. Gómez; A. Serna (comps.). El papel de la memoria en los laberintos de la justicia, la verdad y la reparación (pp. 203-230). Bogotá: Universidad Distrital Francisco José de Caldas-Ipazud.

Savi, V. (2016, marzo 24). De vencedores a víctimas. La memoria institucional del Ejército Argentino. Recuperado de: http://www.revistaharoldo.com.ar/nota.php?id=114.

Stern, S. (2000). Memoria para un nuevo siglo: Chile, miradas a la segunda mitad del siglo xx. Santiago: เom.

Vezzetti, H. (2007). Conflictos de la memoria en la Argentina. Un estudio histórico de la memoria social. En A. Pérotin-Dumon (dir.). Historizar el pasado vivo en América Latina. Recuperado de: http://etica. uahurtado.cl/historizarelpasadovivo/es_contenido.php.

Vinyes, R. (2009). El Estado y la memoria. Gobiernos y ciudadanos frente a los traumas de la historia. Barcelona: RBA.

Wilde, A. (2007). Irruptions of memory: Expressive politics in Chile's transition to democracy. En Journal of Latin American Studies, 31, 473500. [Irrupciones de la memoria: La política expresiva en la transición a la democracia en Chile. En PérotinDumon (dir.). (2007). Historizar el pasado vivo en América Latina. Recuperado de: http://etica. uahurtado.cl/historizarelpasadovivo/es_contenido.php]. 